\title{
NEOPLASIA COLORRETAL ATÉ 40 ANOS - EXPERIÊNCIA EM CINCO ANOS
}

\author{
ELISÂNGELA PLAZAS MONTEIRO ${ }^{1}$, JULIANA BARRETO SALEM ${ }^{1}$, ENZO MARTINS TAGLIETTI $^{1}$, IDBLAN \\ CARVALHO ALBUQUERQUE ${ }^{1}$, GALDINO JOSÉ SITONIO FORMIGA ${ }^{1}$
}

${ }^{1}$ Serviço de Coloproctologia do Hospital Heliópolis, São Paulo, SP, Brasil

MONTEIRO EP, SALEM JB, TAGLIETTI EM, ALBUQUERQUE IC, FORMIGA GJS. Neoplasia colorretal até 40 anos - Experiência em cinco anos. Rev bras Coloproct 2006;26(2):156-161.

RESUMO: Neoplasia colorretal é incomum em menores de 40 anos, ocorrendo numa freqüência de 2,1 a 14,6\%. Neste estudo retrospectivo demonstramos a experiência com pacientes portadores de neoplasia colorretal, submetidos a tratamento cirúrgico ao longo de cinco anos. Dos 453 pacientes operados por neoplasia colorretal no período, 48 (10,6\%) tinham 40 anos ou menos. A faixa etária média foi de 32,5 anos, predominando no sexo masculino (60,4\%). O tempo médio entre o início dos sintomas e o diagnóstico foi de sete meses. História familiar foi positiva em oito (16,7\%), negativa em 28 (58,3\%) e desconhecida em 12 (25\%). Sangramento foi o sintoma mais comum e o reto a principal localização (62,5\%). Cirurgia com intenção curativa foi realizada em 30 casos $(62,5 \%)$. A maioria encontrava-se em estádio III e IV (66,7\%). O seguimento ambulatorial médio foi de 26,7 meses, ocorrendo 12 óbitos neste período.

Descritores: câncer colorretal; idade; pacientes menores de 40 anos; câncer; estadio

\section{INTRODUÇÃO}

A incidência de neoplasia colorretal vêm aumentando nos últimos anos, ocorrendo predominantemente na população idosa, sendo incomum em menores de 40 anos $^{1-3}$. De todos os cânceres colorretais, 2,1 a 14,6\% ocorrem nesta faixa etária ${ }^{4,5}$.

Jovens com câncer colorretal são considerados como tendo pior evolução, porém controvérsias ainda existem quanto às características destes tumores e prognóstico nesta população ${ }^{6}$. Atraso no diagnóstico, estadiamento da doença e agressividade do tumor foram explicações sugeridas para a evolução desfavorável ${ }^{6}$.

Em virtude disto, o nosso objetivo foi demonstrar a experiência do serviço neste grupo de pacientes ao longo de cinco anos.

\section{PACIENTES E MÉTODO}

O estudo retrospectivo descritivo foi realizado no Serviço de Coloproctologia do Hospital Heliópolis, São Paulo - SP, entre janeiro de 2000 a janeiro de 2005, em pacientes com idade menor ou igual a 40 anos,

Trabalho realizado no Serviço de Coloproctologia do Hospital Heliópolis, São Paulo, SP, Brasil

Recebido em 23/05/2006

Aceito para publicação em 09/06/2006 
portadores de neoplasia colorretal e submetidos a tratamento cirúrgico.

Os dados foram coletados através de revisão rigorosa de prontuários. As variáveis analisadas foram idade, sexo, tempo entre início dos sintomas e diagnóstico, história familiar, sintomatologia, localização do tumor, tipo histológico, grau de diferenciação, percentual de mucina, conduta cirúrgica, estadiamento e seguimento ambulatorial.

Todos os pacientes foram submetidos a cirurgia por membros da mesma equipe e o estadiamento realizado de acordo com o sistema TNM do AJCC (American Joint Committee of Cancers). O seguimento foi realizado em ambulatório específico de câncer colorretal.

\section{RESULTADOS}

Dos 453 doentes operados com diagnóstico de câncer colorretal no período, $48(10,6 \%)$ tinham idade menor ou igual a 40 anos. A idade variou de 18 a 40 anos com média de 32,5 anos (Tabela - 1), sendo 29 $(60,4 \%)$ do sexo masculino e $19(39,6 \%)$ do sexo feminino.

O tempo entre o início dos sintomas e admissão hospitalar foi em média de 7 meses. História familiar foi positiva em $8(16,7 \%)$, negativa em $28(58,3 \%)$ e $12(25 \%)$ desconheciam antecedentes. Polipose adenomatosa familiar (PAF) esteve presente em 3 $(6,2 \%)$ casos.

A grande maioria apresentava multiplicidade de sintomas, sendo os mais comuns sangramento $(67,3 \%)$ e alteração do hábito intestinal $(59,6 \%)$.
O reto foi a principal localização, $30(62,5 \%)$ casos, sendo 14 no reto inferior, 11 no médio e 5 no superior. Das 18 (37,5\%) lesões localizadas em cólon, 7 ocorreram no sigmóide, 6 no cólon direito, 4 no descendente e 1 no transverso (Figura -1). Tumores sincrônicos estiveram presentes em apenas um caso, com associação de neoplasia de cólon direito e sigmóide.

Quanto à diferenciação celular, $29(60,4 \%)$ foram moderadamente diferenciados e os indiferenciados perfizeram um total de $12,5 \%$ (Tabela -2). A presença elevada de mucina foi encontrada em 14 $(29,1 \%)$ casos de adenocarcinoma.

Foram realizadas nesta série 84 intervenções cirúrgicas, com média de 1,75 cirurgias por paciente. Cirurgia com intenção curativa foi feita em $30(62,5 \%)$ casos. Ressecção com anastomose primária ocorreu em $23(48 \%)$ e ostomia terminal em $17(35,4 \%)$ casos. A cirurgia mais comum foi retossigmoidectomia anterior seguida pela amputação abdômino-perineal do reto (Tabela - 3). Três doentes foram submetidos a terapia neoadjuvante, 18 a terapia adjuvante e 5 receberam quimioterapia paliativa.

Quanto ao estadiamento pós operatório, oito $(16,7 \%)$ pacientes encontravam-se em estádio I, 7 $(14,6 \%)$ em estádio II, 19 (39,6\%) em estádio III e 13 $(27,1 \%)$ em estádio IV, sendo uma peça cirúrgica negativa após neoadjuvância (Tabela - 4 ).

A média de seguimento ambulatorial foi de 26,7 meses, com perda de oito casos por causa desconhecida. Não ocorreram óbitos no per operatório e $12(25 \%)$ pacientes faleceram neste período (Tabela $-5)$.

Tabela 1 - Distribuição da incidência de câncer

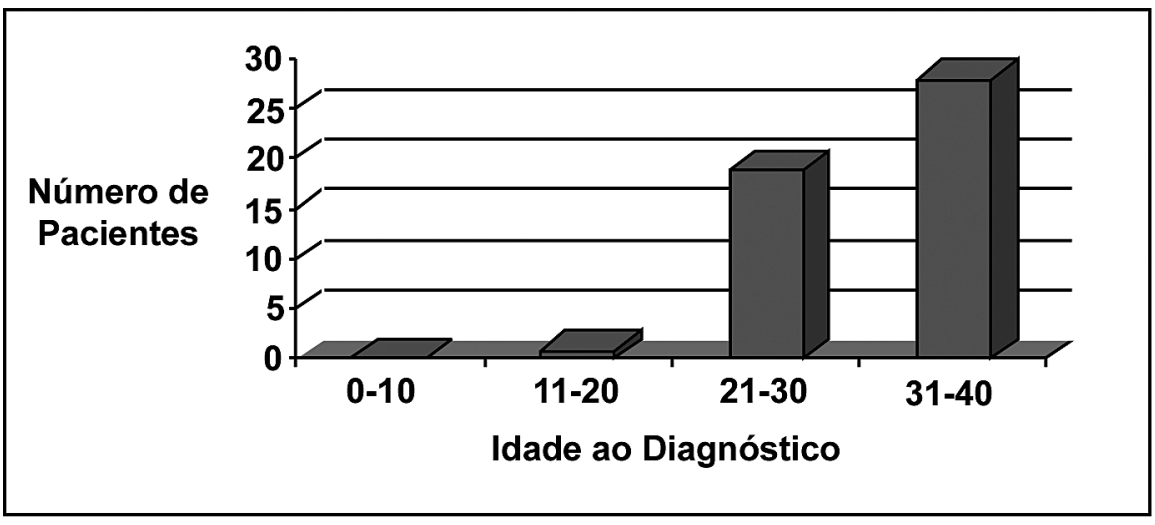




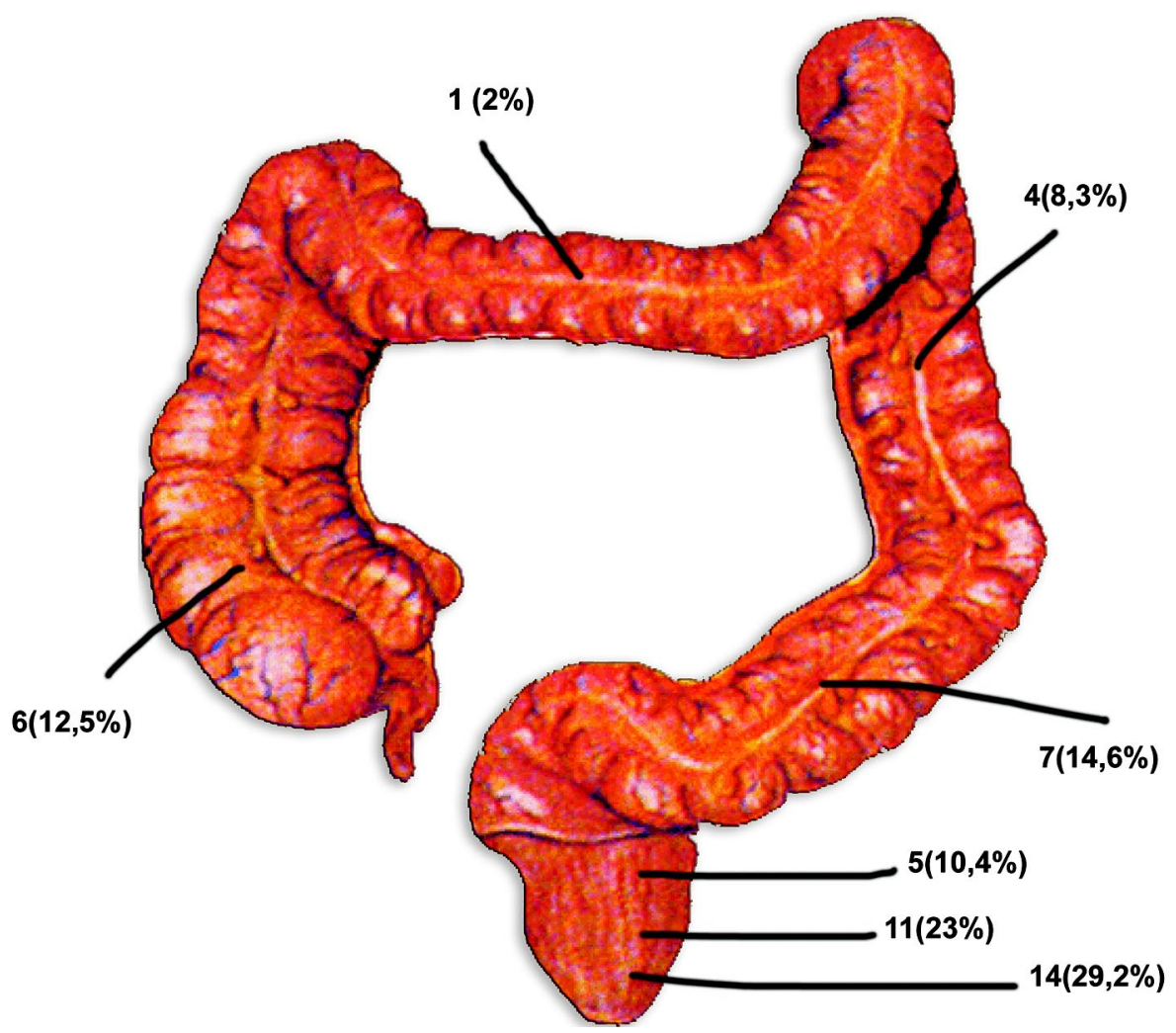

Figura 1 - Localização dos tumores nos cólons e reto.

\section{DISCUSSÃO}

O câncer colorretal é classicamente considerado como doença de indivíduos idosos, com pico de incidência na sexta década de vida ${ }^{7-9}$. Mais de 90\% de todos casos de câncer de cólon e reto ocorrem em pacientes maiores de 50 anos ${ }^{3,10}$. A incidência desta afecção na população jovem (menores de 40 anos) varia de $2,1-14,6 \%^{3-5}$, sendo encontrados em nossa casuística 10,6\%. Em menores de 30 anos a incidência encontrada na literatura é ainda menor (em torno de $1 \%)^{11}$, nesta série ocorreu em $20(4,41 \%)$ casos. A prevalência é ligeiramente maior em homens, com taxas médias de 1,2-3 homens / 1 mulher ${ }^{10}$. Observamos prevalência de 1,47 homens / 1 mulher. O tempo médio entre o início dos sintomas e o diagnóstico varia de 2,3 a 10 meses $^{3,6,10,12}$; no grupo estudado foi de 7 meses, não significando atraso no diagnóstico.

Tabela 2 - Relação entre tipo histológico, grau de diferenciação e percentual de mucina.

\begin{tabular}{|c|c|c|c|c|c|c|}
\hline \multirow[t]{2}{*}{ Tipo Histológico } & \multirow{2}{*}{$\begin{array}{l}\text { Número } \\
\text { de casos }\end{array}$} & \multicolumn{4}{|c|}{ Diferenciação Histológica } & \multirow{2}{*}{$\begin{array}{c}\text { Produçãa de } \\
\text { mucina / cel } \\
\text { em anel de } \\
\text { sinete }\end{array}$} \\
\hline & & $\begin{array}{c}\text { Bem } \\
\text { diferenciados }\end{array}$ & $\begin{array}{l}\text { Moderadamente } \\
\text { diferenciados }\end{array}$ & Indiferenciados & $\begin{array}{c}\text { Sem } \\
\text { especificação }\end{array}$ & \\
\hline Adenocarcinoma & 48 & $7(14,6 \%)$ & $29(60,4 \%)$ & $6(12,5 \%)$ & $6(12,5 \%)$ & $14(29,2 \%)$ \\
\hline
\end{tabular}


Tabela 3 - Intervenções cirúrgicas.

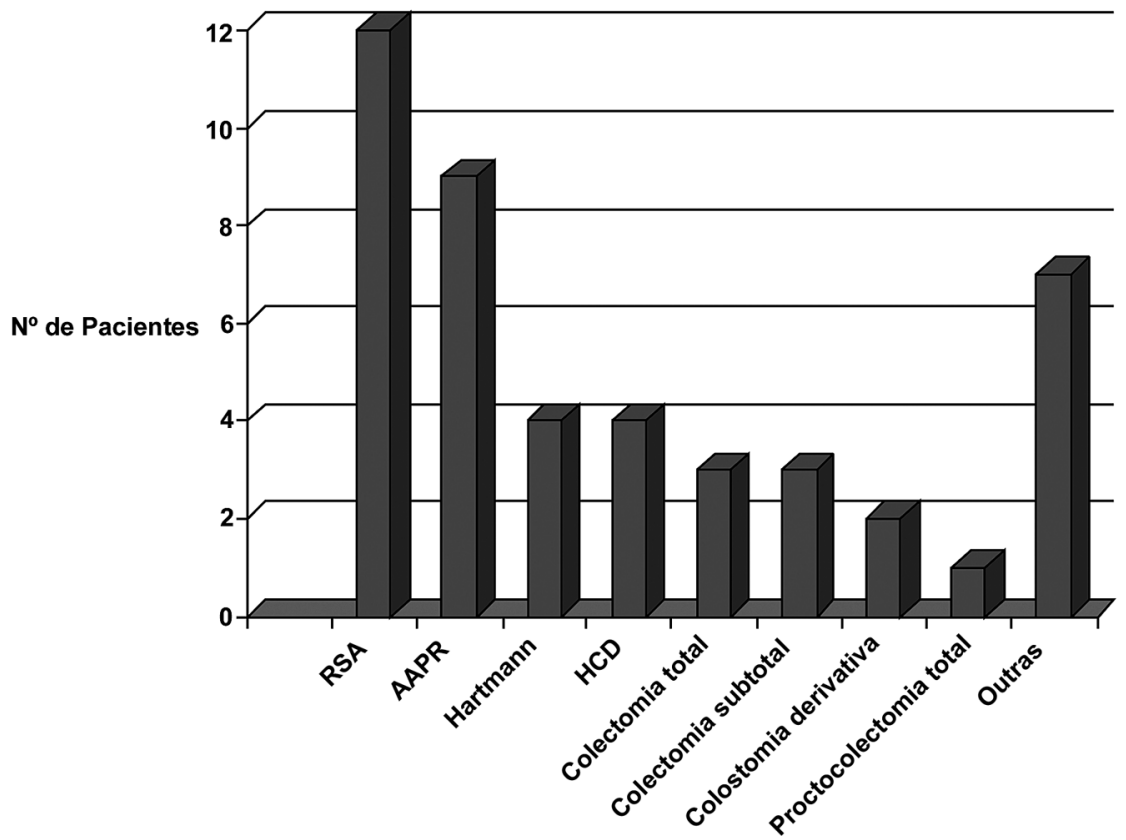

Tabela 4 - Distribuição segundo o estádio.

\begin{tabular}{lc}
\hline Estadiamento TNM & $\begin{array}{c}\text { Número de pacientes } \\
\text { / porcentagem }\end{array}$ \\
\hline Estádio I & $8(16,7 \%)$ \\
Estádio II & $7(14,6 \%)$ \\
Estádio III & $19(39,6 \%)$ \\
Estádio IV & $13(27,1 \%)$ \\
\hline
\end{tabular}

* 1 peça cirúrgica negativa pós neoadjuvância (2\%)
A história familiar, bem como a associação sindrômica do HNPCC (câncer colorretal hereditário não polipóide) e FAP (polipose adenomatosa familiar), é pouco observada, talvez devido às dificuldades de rastreamento $^{3}$. Lee PY et al $^{6}$ observaram $39 \%$ de fatores de risco associados, como doença inflamatória intestinal, pólipos e FAP, não encontrando casos de HNPCC. Os autores afirmaram ainda que traços de FAP estiveram associados a uma incidência de 58\%

Tabela 5 - Óbitos durante o seguimento.

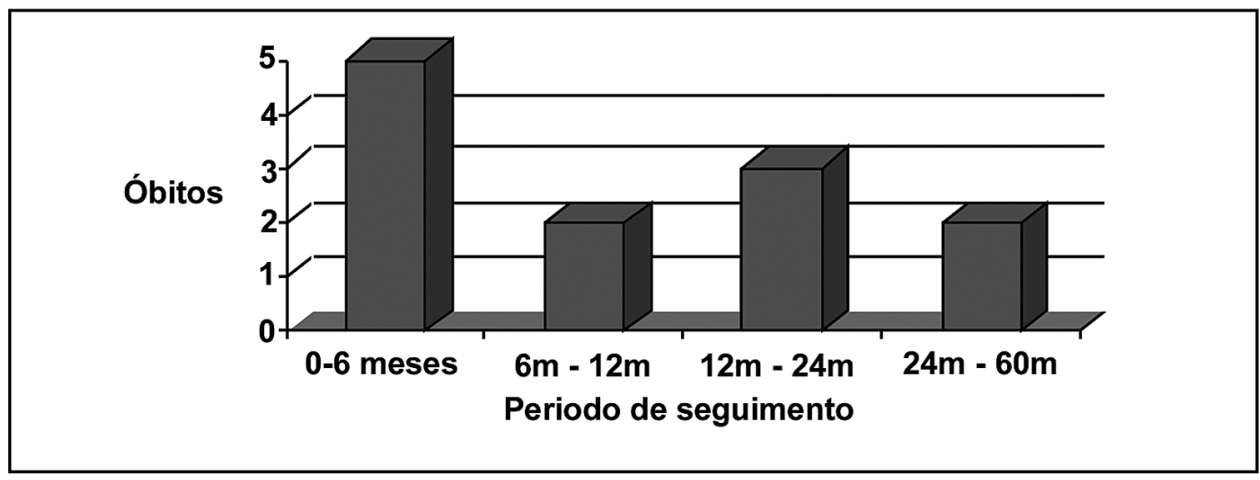


de câncer colorretal antes dos 28 anos. Liang JT et al ${ }^{13}$ não encontraram maior incidência de história familiar quando compararam jovens e idosos. mucinoso, ao passo que Lupinacci RM et $\mathrm{al}^{5}$ encontraram proporção de $28,8 \%$, o que é semelhante ao encontrado neste estudo (29,2 \% dos adenocarcinomas). Comparada à população geral, 10-20\% dos adenocarcinomas são mucinosos $^{27}$. Os sintomas predominantes, nas séries analisadas, foram dor abdominal, sangramento e alteração do hábito intestinal ${ }^{3-6,9,14,15}$. A multiplicidade de sintomas foi relacionada a pior prognóstico ${ }^{5,16}$. Prognóstico melhor tem sido observado em tumores com localização em cólon direito ${ }^{17}$, e, quanto mais distal a localização tumoral, pior o prognóstico ${ }^{17}$. Observa-se maior predominância de envolvimento do reto ${ }^{5,6,14}$, assim como encontrado por nós. Tumores sincrônicos ocorreram em $2 \%$ de nossos pacientes, taxa inferior à encontrada na literatura consul$\operatorname{tada}^{6,18,19}$.Numerosos estudos têm relatado que ao diagnóstico pacientes jovens encontram-se em estádio avançado, associado a um comportamento bioloicamente agressivo e distribuição tumoral diferente, tem conferido pior prognóstico ${ }^{5,11,20-22}$. Em nossa série, $66,7 \%$ encontravam-se em estádio avançado (III e IV) no momento do diagnóstico, semelhante ao encontrado na literatura. Em suas séries, Lee PY et $\mathrm{al}^{6}$ e Parramore JB et $\mathrm{al}^{3}$ encontraram incidência de doença avançada em $71 \%$ e $78 \%$ respectivamente. A incidência em doentes idosos, encontrada por outros autores, foi inferior ao grupo jovem, variando de 53 a $57 \%{ }^{23}$. Segundo alguns autores, o estadiamento no momento do diagnóstico foi o melhor indicador prognóstico ${ }^{24,25}$. A maior proporção de doença avançada neste grupo vêm sendo atribuída a atraso no diagnóstico ou demora na procura ao médico ${ }^{19,26}$. Adenocarcinoma mucinoso, em qualquer grau de diferenciação, é considerado como fator localmente agressivo e de pior prognóstico, ${ }^{9,15}$. Kam MH et al $^{2}$ descreveram incidência de $18 \%$ de adenocarcinoma mucinoso, ao passo que Lupinacci RM et $\mathrm{al}^{5}$ encontraram proporção de $28,8 \%$, o que é semelhante ao encontrado neste estudo $(29,2 \%$ dos adenocarcinomas). Comparada à população geral, 10$20 \%$ dos adenocarcinomas são mucinosos ${ }^{27}$.Cirurgia com intenção curativa foi realizada em $62,5 \%$ dos casos, com ausência de óbitos per operatórios,. indicando que tais pacientes toleram bem complicações cirúrgicas, dado compatível com a literatura consultada ${ }^{15}$. Durante seguimento médio de 26,7 meses, 36 a $50 \%$ dos pacientes evoluíram com recidiva neoplásica $^{13}$. No mesmo período ocorreram 12 (25\%) óbitos, seja por doença inicialmente avançada ou por recidiva tumoral.

\section{CONCLUSÕES}

Apesar de idade jovem sugerir ausência de neoplasia maligna, em nossa casuística, não foi observado retardo no diagnóstico em relação ao início dos sintomas, quando comparado ao tempo de diagnóstico em população mais idosa, encontrado na literatura. Isto sugere este fator como não sendo responsável pela maior agressividade dos tumores nesta faixa etária.

Ainda assim, a maioria dos pacientes encontrava-se em estádio mais avançado (III e IV), não sendo encontrada nenhuma variável relacionada a este achado.

Um elevado índice de suspeita, com análise cuidadosa da história familiar, aliada aos avanços da genética, podem influenciar positivamente no rastreamento e diagnóstico mais precoce destes pacientes.

SUMMARY: Colorectal cancer is an unusual disease in patients under 40 years of age, occurring with a frequency of 2.1 to $14.6 \%$. In this retrospective study, we reviewed all patients with colorectal cancer under 40 years of age, submitted to surgery over a 5 year period. From all the 453 patients operated on with this disease at that period $48(10.6 \%)$ were 40 years old or less. The average age was 32.5 years and the majority of patients were male $(60.4 \%)$. The average time between the appearance of the symptoms and the diagnosis was 7 months. Family history was positive in eight (16.7\%), negative in $28(58.3 \%)$ and unknown in $12(25 \%)$ patients. Rectal bleeding was the most important symptom and the rectum was the main location of the disease $(62.5 \%)$. Thirty patients (62.5\%) were submitted to curative surgery. The majority of patients $(66.7 \%)$ presented the disease in advanced stages (III and IV). The average follow -up was 26.7 months with 12 deaths occurring in this period.

Key words: colorectal cancer; age; patients under 40 years; cancer; stage 


\section{REFERÊNCIAS BIBLIOGRÁFICAS}

1. Brown RA, Rode H, Millar AJW, Sinclair-Smith C, Cywes S. Colorectal carcinoma in children. J Ped Surg 1992; 27(7):919-921.

2. Kam MH, Eu KW, Barben CP, Seow Choen F. Colorectal cancer in the young: a 12-year review of patients 30 years or less. Colorectal Dis 2004; 6(3):191-194.

3. Parramore JB, Wei JP, Yeh KA. Colorectal cancer in young patients under forty: presentation and outcome. Am Surg 1998; 64(6):563-568.

4. Lupinacci RM, Campos FGCM, Araújo SEA, Imperiale AR, Seid VE, Habr-Gama A et al. Análise comparativa das características clínicas, anátomo-patológicas e sobrevida entre pacientes com câncer colo-retal abaixo e acima de 40 anos de idade. Rev bras Coloproct 2003; 23(3): 155-162.

5. Smith C, Butler JA. Colorectal cancer in patients younger than 40 years of age. Dis Colon Rectum 1989; 32(10):843846.

6. Lee PY, Fletcher WS, Sullivan ES, Vetto JT. Colorectal cancer in young patients: characteristics and outcome. The American Surgeon 1994; 60(8):607-612.

7. Chantada GL, Perelli VB, Lombardi MG, Amaral D, Cascallar $\mathrm{D}$, Scopinaro $\mathrm{M}$ et al. Colorectal carcinoma in children, adolescents, and young adults. J Pediatric Hematol Oncol 2005; 27(1):39-41.

8. Frizis H, Papadopoulos A, Akritids G, Frizis HR, Hatzitheoharis G. Are there any differences in colorectal cancer between young and elderly patients? Tech Coloproct 2004; 8S:147-148.

9. McGahren ED 3rd, Mills SE, Wilhelm MC. Colorectal carcinoma in patients 30 years of age and younger. Am Surg 1995; 61(1):78-82.

10. Drumond CA, Ferro RAF, Nogueira AMF, Profeta da Luz MM, Conceição AS, Silva RG et al. Câncer colorretal em pacientes com idade inferior a 30 anos. Rev bras Coloproct 2003; 23(3):147-154.

11. Gallagher EG, Zeigler MG. Rectal carcinoma in the second and third decades of life. Am J Surg 1972; 124:655-658.

12. Marble K, Banerjee S, Greenwald L. Colorectal carcinoma in young patients. J Surg Oncol 1992; 51:179-182.

13. Liang JT, Huang KC, Cheng AL, Jeng YM, Wu MS, Wang SM . Clinicopathological and molecular biological features of colorectal cancer in patients less than 40 years of age. Br J Surg 2003; 90:205-214.

14. Taylor MC, Pounder D, Ali-Ridha NH, Bodurtha A, MacMullin EC. Prognostic factors in colorectal carcinoma of young adults. Can J Surg 1988; 31(3):150-153.
15. Walton Jr WW, Hagihara PF, Griffen Jr WO. Colorectal adenocarcinoma in patients less than 40 years old. Dis Colon Rectum 1975; 19(6):529-534.

16. Polissar L, Sim D, Francis A. Survival of colorectal cancer in relation to duration of symptoms and other prognostic factors. Dis Colon Rectum 1981; 24(5):364-369.

17. Alici S, Aykan NF, Sakar B, Bututlar G, Kaytan E, Topuz E. Colorectal cancer in young patients: characteristics and outcome. Tohoku J Exp Med 2003; 199(2):85-93.

18. Enker WE, Dragacevic S. Multiple carcinomas of the large bowel. Ann Surg 1978; 187(1): 8-11.

19. MacGillivray DC, Swartz SE, Robinson AD, Cruess DF, Smith LE. Adenocarcinoma of the colon and rectum in patients less than 40 years of age.Surg Gynecol Obstet 1991;172(1):1-7.

20. Isbister WH, Fraser J. Large-bowel cancer in the young: a national survival study. Dis Colon Rectum 1990; 33(5):363366.

21. O’Connell JB, Maggard MA, Liu JH, Etzioni DA, Ko CY. Are survival rates different for young and older patients with rectal cancer ? Dis Colon Rectum 2004; 47(12):2064-2069.

22. Rao BN, Pratt CB, Fleming ID, Dilawari RA, Green AA, Austin BA. Colon carcinoma in children and adolescents. Cancer 1985; 55(6):1322-1326.

23. Frey CM, McMillen MM, Cowan CD. Representativeness of the surveillance, epidemiology, and end results program data: Recent trends in cancer mortality rates. JNCI 1992; 84:872877.

24. Adloff M, Arnaud JP, Schloegal M, Thibaud D, Bergamaschi R. Colorectal cancer in patients under 40 years of age. Dis Colon Rectum 1986; 29(5):322-325.

25. Safford KL, Spebar MJ, Rosenthal D. Review of colorectal cancer in patients under age 40 years. Am J Surg 1981; 142:767-769.

26. Pitluk H, Poticha SM. Carcinoma of the colon and rectum in patients less than 40 years of age. Surg. Gynecol Obstet 1983; 157:335-337.

27. Symons DA, Vicfery Jr AL. Mucinous carcinoma of the colon and rectum. Cancer 1976; 37(4):1891-1900.

Endereço para correspondência: GALDINO JOSÉ SITONIO FORMIGA

Hospital Heliópolis - Serviço de Coloproctologia

Rua Cônego Xavier, 276 - Vila Heliópolis

04.231-030 - São Paulo-SP

Tel: (11) 2274-7600 (Ramal 244)

E-mail: eliplazas@terra.com.br 